\title{
Magneto-enhanced electro-thermal conversion performance
}

\author{
Shifang $\mathrm{Ma}^{1,2 \dagger}$, Cuncheng $\mathrm{Li}^{1 \dagger}$, Wenjun Cui ${ }^{1,3}$, Xiahan Sang ${ }^{1,3^{*}}$, Ping Wei ${ }^{1,3}$, Wanting Zhu ${ }^{1}$, \\ Xiaolei Nie ${ }^{1}$, Fu-Hua Sun ${ }^{1,2}$, Wenyu Zhao ${ }^{1 *}$ and Qingjie Zhang ${ }^{1}$
}

\begin{abstract}
Synergistically regulating carrier and phonon transport on the nanoscale is extremely difficult for all thermoelectric (TE) materials without cage structures. Herein $\mathrm{BaFe}_{12} \mathrm{O}_{19} / \mathrm{Bi}_{2} \mathrm{Te}_{2.5} \mathrm{Se}_{0.5}$ thermoelectromagnetic nanocomposites are designed and synthesized as a benchmarking example to simultaneously tailor the transport properties on the nanoscale. A magneto-trapped carrier effect induced by $\mathrm{BaFe}_{12} \mathrm{O}_{19}$ hard-magnetic nanoparticles (NPs) is discovered, which can lower the carrier concentration of n-type $\mathrm{Bi}_{2} \mathrm{Te}_{2.5} \mathrm{Se}_{0.5}$ matrix by $16 \%$, and increase the Seebeck coefficient by $16 \%$. Meanwhile, $\mathrm{BaFe}_{12} \mathrm{O}_{19} \mathrm{NPs}$ provide phonon scattering centers and reduce the thermal conductivity by $12 \%$. As a result, the $Z T$ value of the nanocomposites is enhanced by more than $25 \%$ in the range of $300-450 \mathrm{~K}$, and the cooling temperature difference increases by $65 \%$ near room temperature. This work greatly broadens the commercial application potential of $\mathbf{n}$ type $\mathrm{Bi}_{2} \mathrm{Te}_{2.5} \mathrm{Se}_{0.5}$, and demonstrates magneto-trapped carrier effect as a universal strategy to enhance the electro-thermal conversion performance of TE materials with high carrier concentration.
\end{abstract}

Keywords: thermoelectromagnetic nanocomposite, thermoelectric material, magnetic nanoparticles, magneto-trapped carrier effect, electro-thermal conversion performance

\section{INTRODUCTION}

Thermoelectric (TE) applications in cooling, heating, power generation, and waste heat recovering pose great opportunities and challenges to further increase the electro-thermal conversion performance of TE materials [1]. High TE conversion efficiency requires large dimensionless TE figure of merit defined as $Z T=\alpha^{2} \sigma T / \kappa$, where high Seebeck coefficient $\alpha$, large electrical con- ductivity $\sigma$, and low thermal conductivity $\kappa$ (including carrier thermal conductivity $\kappa_{\mathrm{E}}$ and lattice thermal conductivity $\kappa_{\mathrm{L}}$ ) are desired. Therefore, simultaneously regulating carrier and phonon transport plays a fundamental role in enhancing TE performance and discovering new TE materials [2]. Until now, synergistically regulating carrier and phonon transport on the same scale is extremely difficult for all TE materials without cage structures. For those TE materials with cage structures such as skutterudite and clathrate, one can use different atoms to fill the cages to optimize the band structure and to provide scattering centers for phonon, realizing simultaneous regulation of carrier and phonon transport on the atomic scale [3-6]. However, for all other TE materials, one can only separately tune electric transport properties by band engineering on the atomic scale [7-10], and/or thermal transport properties by phonon engineering on the nanoscale [11-13]. It is extremely important to develop new methods for TE materials without cage structure to synergistically control electron and phonon transport on the same scale.

$\mathrm{Bi}_{2} \mathrm{Te}_{3}$-based alloys are a kind of low-temperature $\mathrm{TE}$ materials with best performance in the range of room temperature to $200^{\circ} \mathrm{C}$, and possess great potential in commercial applications [14-16]. Near room temperature, the $Z T$ values of p-type $\mathrm{Bi}_{2} \mathrm{Te}_{3}$-based materials have reached around 1.4 through various methods [17-19], while the $Z T$ values for n-type $\mathrm{Bi}_{2} \mathrm{Te}_{3}$-based alloy have been wandering around 0.7 in the last two decades $[15,20,21]$. The main reason is that $n$-type $\mathrm{Bi}_{2} \mathrm{Te}_{3}$-based alloys typically have high carrier concentrations from the donor-like effect during sample preparation [22,23], which lowers $\alpha$ and significantly limits their conversion

\footnotetext{
${ }^{1}$ State Key Laboratory of Advanced Technology for Materials Synthesis and Processing, Wuhan University of Technology, Wuhan 430070, China

${ }^{2}$ Foshan Xianhu Laboratory of the Advanced Energy Science and Technology Guangdong Laboratory, Foshan 528000, China

${ }^{3}$ Nanostructure Research Center, Wuhan University of Technology, Wuhan 430070, China

† These authors contributed equally to this work.

* Corresponding authors (emails: wyzhao@whut.edu.cn (Zhao W); xhsang@whut.edu.cn(Sang X))
} 
performance. Therefore, for n-type $\mathrm{Bi}_{2} \mathrm{Te}_{3}$ alloys, a revolutionary strategy is critically needed to regulate the carrier concentration for the enhancement of the $Z T$ values.

Recently, it was discovered that incorporating magnetic nanoparticles (NPs) in TE material can simultaneously regulate carrier and phonon transport on the nanoscale [24-27]. Since then, magnetism has been regarded as a new degree of freedom to tailor electron and phonon transport in TE materials [26,28-30]. Now the electron multiple scattering effect induced by superparamagnetism has been widely used not only in the low-temperature $\mathrm{Bi}_{2} \mathrm{Te}_{3}$ alloys [31,32], but also in the middle-temperature TE materials with best TE performance in the range of 200-500 ${ }^{\circ} \mathrm{C}$, such as $\mathrm{Cu}_{2} \mathrm{ZnSnS}_{4}$ [33], and $\mathrm{Ba}_{0.3} \mathrm{In}_{0.3} \mathrm{Co}_{4} \mathrm{Sb}_{12}$ [25]. The electron repository effect, which traps electrons below Curie temperature and releases electrons above Curie temperature, has been discovered to be effective for middle-temperature TE materials such as $\mathrm{Ba}_{0.3} \mathrm{In}_{0.3} \mathrm{Co}_{4^{-}}$ $\mathrm{Sb}_{12}$ [24] and half-Heusler alloys [26]. However, this novel effect has not been applied to the low-temperature TE materials because their working temperature is generally lower than the Curie temperature of magnetic NPs.

Herein, a series of thermoelectromagnetic nanocomposites have been proposed by incorporating $\mathrm{BaFe}_{12^{-}}$ $\mathrm{O}_{19} \mathrm{NPs}$ (BaM-NPs) into n-type $\mathrm{Bi}_{2} \mathrm{Te}_{2.5} \mathrm{Se}_{0.5}$ (BiTeSe) as a new degree of freedom to synergistically regulate electron and phonon transport on the nanoscale. The high Curie temperature $(669-673 \mathrm{~K})$ of hard-magnetic BaM-NPs ensures that their local magnetic field could regulate carrier transport in the working temperature range (300-500 K) of BiTeSe. The magneto-trapped carrier effect induced by BaM-NPs is discovered to lower the carrier concentration and increase the Seebeck coefficient of the BiTeSe matrix. Additionally, BaM-NPs provide phonon scattering centers and reduce the thermal conductivity. Consequently, introducing BaM-NPs into ntype BiTeSe matrix provides a new strategy to synergistically regulate electron and phonon transport on the nanoscale, especially for those TE materials whose high carrier concentrations are difficult to be lowered.

\section{EXPERIMENTAL SECTION}

High-purity $\mathrm{Bi}(5 \mathrm{~N}), \mathrm{Te}(5 \mathrm{~N})$ and $\mathrm{Se}(5 \mathrm{~N})$ powders were weighed according to the nominal compositions of n-type $\mathrm{Bi}_{2} \mathrm{Te}_{2.5} \mathrm{Se}_{0.5}$ (BiTeSe). To ensure the composition homogeneity, the powders were mixed and ground in a mortar, sealed in an evacuated quartz tube at $0.1 \mathrm{~Pa}$, and then melted at $1073 \mathrm{~K}$. The obtained ingots were manually crushed into fine powders and then sifted through 120- mesh sieve to obtain matrix powders with sizes less than $125 \mu \mathrm{m}$. The micron-sized BaM powders were prepared by combining co-precipitation and high-temperature annealing in air, and then the as-prepared powders were ball-milled for $24 \mathrm{~h}$ and let stand for $12 \mathrm{~h}$ in ethanol to obtain BaM-NPs. To prepare $x \mathrm{BaM}-\mathrm{NP} / \mathrm{BiTeSe}$ nanocomposites, different contents of BaM-NPs were weighed using $x=0,0.15,0.30,0.45$ and $0.60 \mathrm{wt} \%$, and then mixed with the as-prepared BiTeSe powders via ultrasonic dispersion. These $\mathrm{BaM} / \mathrm{Bi}_{2} \mathrm{Te}_{2.5} \mathrm{Se}_{0.5}$ powders were placed in a $\phi 15 \mathrm{~mm}$ graphite die and sintered into bulk materials at $723 \mathrm{~K}$ for $5 \mathrm{~min}$ under the uniaxial stress of $50 \mathrm{MPa}$.

The phase compositions of the BiTeSe matrix and $x \mathrm{BaM}-\mathrm{NPs} / \mathrm{BiTeSe}$ nanocomposites were determined using powder X-ray diffraction (XRD) with $\mathrm{Cu} \mathrm{Ka}$ radiation $(\lambda=0.15418 \mathrm{~nm})$. The influence of NPs on the fracture surfaces of the nanocomposites was characterized by a field emission scanning electron microscope (FESEM, Hitachi SU-8020, Japan). The chemical compositions and the distribution of BaM-NPs embedded into the nanocomposites were investigated by electron probe $\mathrm{mi}$ croanalysis (EPMA, JEOL, JXA-8230) and double spherical aberration-corrected scanning transmission electron microscope (STEM, Titan Cubed Themis G2 300).

Due to the intrinsic anisotropy of the BiTeSe crystal structure, the transport properties of all samples were measured along the in-plane direction. The sintered bulk samples were cut into bars (about $2.5 \mathrm{~mm} \times 3.5 \mathrm{~mm} \times$ $11.5 \mathrm{~mm}$ ) for simultaneously measuring the $\sigma$ and $\alpha$ using a standard four-probe method (Sinkuriko, ZEM-3) under helium atmosphere. The $\kappa$ was calculated from $\kappa=\lambda d C_{\mathrm{p}}$, where $d$ is the bulk density determined by the Archimedes method, $C_{\mathrm{p}}$ is the specific heat capacity measured using a differential scanning calorimeter (TA instruments, Q20), and $\lambda$ is the thermal diffusivity coefficient tested by the laser flash diffusivity method using a Netzsch LFA-457 system. The $\kappa_{\mathrm{L}}$ was obtained by subtracting the electrical contribution from $\kappa$ by using the equation $\kappa_{\mathrm{L}}=\kappa-\kappa_{\mathrm{E}}$. $\kappa_{\mathrm{E}}$ is expressed by the WiedemannFranz $\kappa_{\mathrm{E}}=\sigma L T$. The high temperature Hall coefficient $\left(R_{\mathrm{H}}\right)$ was measured from 300 to $500 \mathrm{~K}$ by a self-made TE and magnetic performance testing system. After $R_{\mathrm{H}}$ was obtained, the $n$ and $\mu_{\mathrm{H}}$ were calculated according to the equations $n=1 /\left(R_{\mathrm{H}} e\right)$ and $\mu_{\mathrm{H}}=\sigma / n e$, where $e$ is the electron charge. The magnetic properties were measured using VersaLab (Quantum Design). BaM/ $\mathrm{Bi}_{2} \mathrm{Te}_{2.5} \mathrm{Se}_{0.5}$ single-leg cooling devices with a dimension of $2.5 \mathrm{~mm} \times 3.5 \mathrm{~mm} \times 11.0 \mathrm{~mm}$ were fabricated by welding two copper electrodes at both ends of the bulk TE materials. A direct power supply (Gwinstek, GPD-2303S) 
was used to provide the working currents. Temperatures at both ends were measured using a high-precision T-type thermocouple (OMEGA Engineering INC.) and infrared imager (Ti450, Fluke), and the data were recorded by the acquisition module and LabVIEW program.

\section{RESULTS AND DISCUSSION}

\section{Microstructure characterization}

A series of $\mathrm{n}$-type $x \mathrm{BaM}-\mathrm{NP} / \mathrm{BiTeSe}$ thermoelectromagnetic nanocomposites $(x=0,0.15 \%, 0.30 \%, 0.45 \%$, $0.60 \%$, named MNC00, MNC15, MNC30, MNC45, MNC60, respectively) were prepared by ultrasonic dispersion and spark plasma sintering. The phase compositions of the BiTeSe matrix and BaM-NPs/BiTeSe nanocomposites were characterized using XRD. All the main diffraction peaks of the BiTeSe matrix can be indexed to the crystal structure of $\mathrm{Bi}_{2} \mathrm{Te}_{3}$ (JCPDS 15-0813) (Fig. 1a). The BiTeSe (10I므) and (1120) diffraction peaks (Fig. 1b) of all the nanocomposites show negligible displacement, suggesting that BaM-NPs do not change the crystal structure of BiTeSe. Although the characteristic diffraction peaks of $\mathrm{BaM}$ are not detected in the nanocomposites due to the detection limit of XRD (about $1 \%)$, the existence of $\mathrm{BaM}$ is confirmed by EPMA (Fig. S1).

Fig. 1c, d show the FESEM images of the fractured surfaces of the BiTeSe matrix (MNC00) and 0.30\% BaM$\mathrm{NPs} / \mathrm{BiTeSe}$ (MNC30) nanocomposite. BaM-NPs are not observed at the grain boundaries of MNC00 (Fig. 1c). BaM-NPs with diameter of $\sim 100 \mathrm{~nm}$ are randomly distributed at the interfaces and grain boundaries of BiTeSe in $x$ BaM-NPs/BiTeSe (Fig. 1d), which can increase the phonon scattering and reduce the thermal conductivity [19].

The microstructures and chemical compositions of BaM-NPs in MNC30 were further characterized using STEM (Fig. 2). Low-magnification STEM image (Fig. 2a) shows that BaM-NPs (dark contrast) with an average diameter of roughly $100 \mathrm{~nm}$ are embedded in the BiTeSe matrix. Energy dispersive X-ray spectroscopy (EDX) elemental mapping (Fig. 2b) demonstrates that the region with bright contrast is BiTeSe matrix and the dark-contrast region is BaM-NPs. Atomic-resolution high-angle annular dark field (HAADF)-STEM image acquired from the BiTeSe matrix (Area 1 in Fig. 2a) matches well with
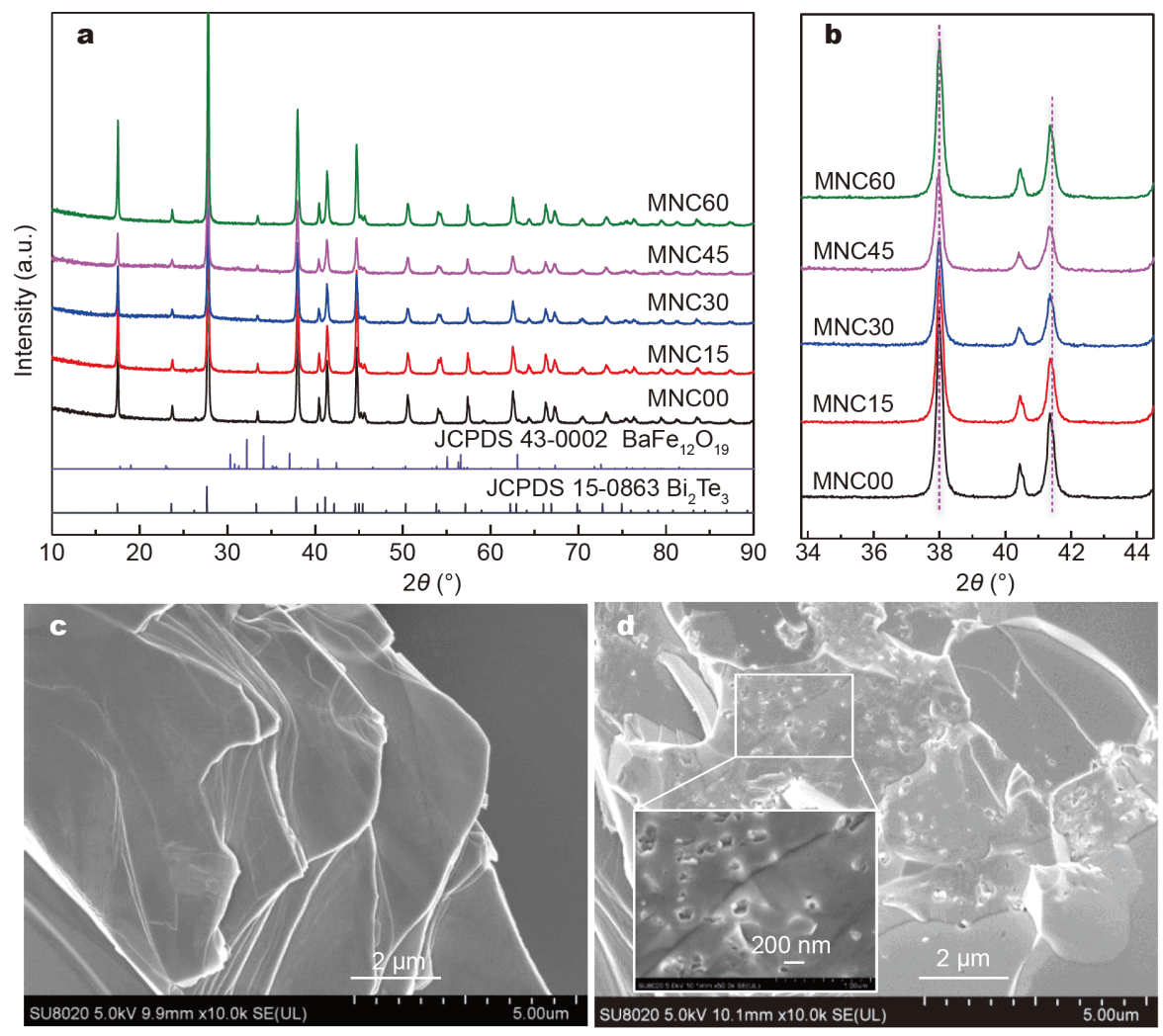

Figure 1 Microstructural characterizations using XRD and FESEM. (a, b) XRD patterns of BiTeSe matrix and $x \mathrm{BaM}-\mathrm{NPs} / \mathrm{BiTeSe}$ thermoelectromagnetic nanocomposite materials. (c, d) FESEM images for fracture surfaces of (c) MNC00 and (d) MNC30. 


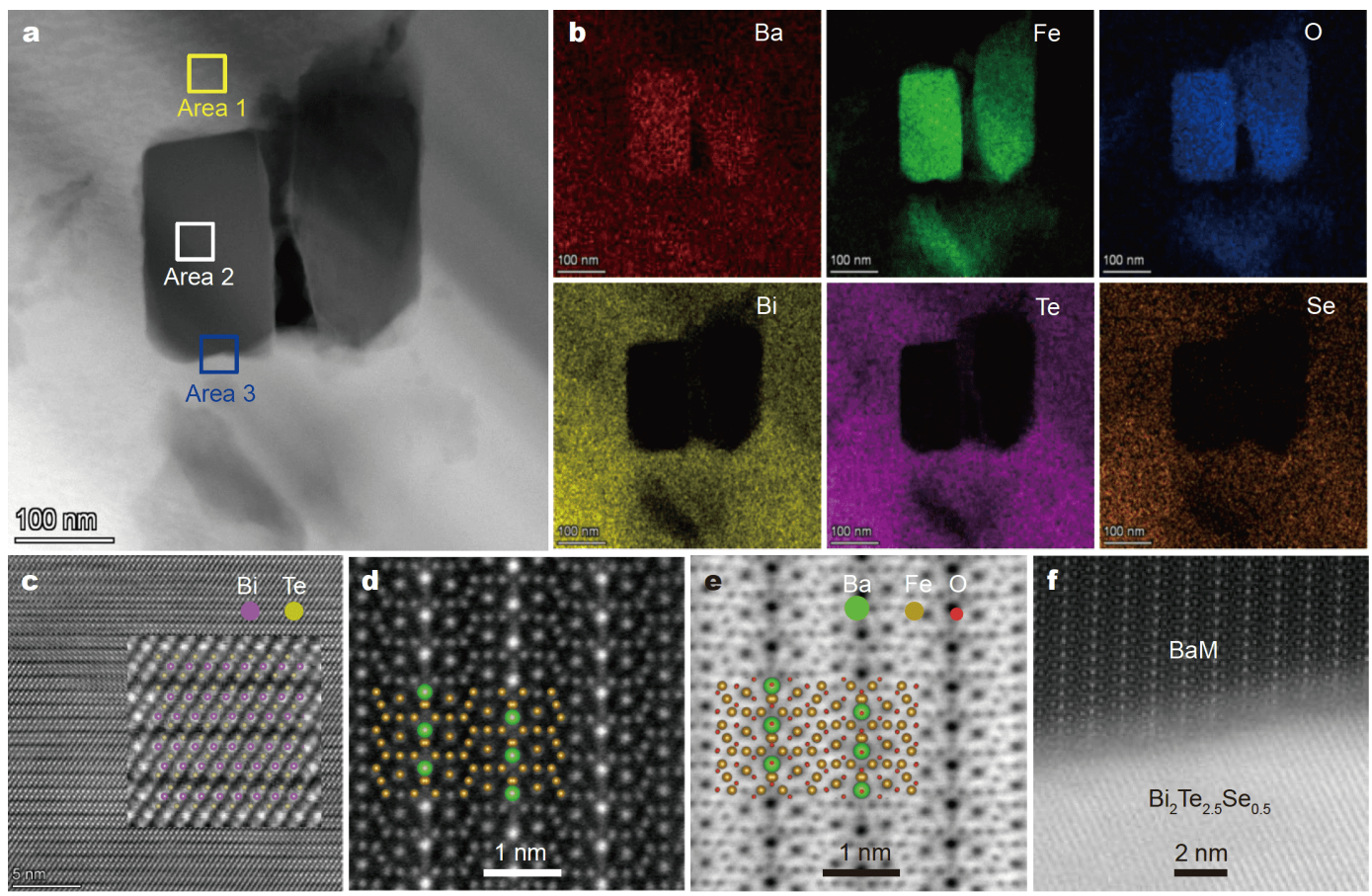

Figure 2 Atomic structure analyses using HAADF STEM and EDS. (a) HAADF-STEM image of MNC30; (b) STEM-EDX elemental mapping images of $\mathrm{Ba}, \mathrm{Fe}, \mathrm{O}, \mathrm{Bi}, \mathrm{Te}$, Se of (a); (c) enlarged HRTEM image of Area 1 in (a), and the inset is the corresponding atomic resolution HAADF-STEM image; (d) atom-resolution HAADF-STEM dark image from Area 2 in (a); (e) atom-resolution HAADF-STEM bright image from Area 2 in (a); (f) enlarged HRTEM image of Area 3 in (a).

the projected crystal structure of $\mathrm{Bi}_{2} \mathrm{Te}_{3}$ along [2I10] zone axis (Fig. 2c). The atom-resolution HAADF-STEM and annular bright field (ABF) STEM images (Fig. 2d, e) from Area 2 in Fig. $2 \mathrm{a}$ are consistent with the atomic model of $\mathrm{BaM}$ along the [2110] zone axis. Fig. 2f shows a typical interface between the BiTeSe matrix and BaM-NPs, without formation of defects at the interface or interfacial reaction. Such interface can increase phonon scattering, while it has minimal influence on the electric transport properties [34].

\section{Built-in magnetic field in $x$ BaM-NPs/BiTeSe}

As BiTeSe matrix is diamagnetic and BaM-NPs are ferromagnetic, it is important to characterize the overall magnetic properties of the nanocomposites to understand their influence on the carrier transport. BaM has a hexagonal crystal structure, and its magnetism comes from the $\mathrm{Fe}^{3+}$ ions (high spin) at $\mathrm{O}^{2-}$ polyhedron interstitial positions. The room-temperature hystersis loop of BaMNPs shows a ferromagnetism behavior with large saturation magnetization $\left(9.14 \mu_{\mathrm{B}}\right.$ f.u. $\left.^{-1}\right)$, remanence $\left(4.29 \mu_{\mathrm{B}}\right.$ f.u. $\left.^{-1}\right)$, and coercivity (3443 Oe) (Fig. 3a). On the other hand, the BiTeSe matrix exhibits typical a dia- magnetism behavior with negative susceptibility (Fig. 3b) [35]. Due to the strong ferromagnetism of BaM-NPs, a small amount of BaM-NPs added in the BiTeSe matrix is sufficient to convert the nanocomposite to ferromagnetic, and the saturation magnetization and the remanence are proportional to the BaM-NPs content (Fig. 3c). For example, the hysteresis loop of MNC30 nanocomposite looks similar to that of the BaM-NPs, with a saturation magnetization of $2.8 \times 10^{-2} \mu_{\mathrm{B}}$ f.u. $^{-1}$ and a remanence of $1.3 \times 10^{-2} \mu_{\mathrm{B}}$ f.u. $^{-1}$, which scales well with 9.14 and $4.29 \mu_{\mathrm{B}}$ f.u. $^{-1}$, given that only $0.3 \mathrm{wt} \%$ BaM-NPs were added to the nanocomposite. The coercivity of all the nanocomposites is $3445 \mathrm{Oe}$, which agrees well with the coercivity of pure BaM-NPs.

Conventionally, magnetic doping such as $\mathrm{Mn}-$ and $\mathrm{Cr}$ doping is a typical approach to introduce remnant internal magnetic field in $\mathrm{Bi}_{2} \mathrm{Te}_{3}$-based materials. However, magnetic doping tends to introduce point defects, change the lattice constants and band structure, influence the carrier concentration and carrier mobility, and complicate the interpretation of electric transport properties. On the contrary, a small amount $(0.15 \mathrm{wt} \%)$ of BaM-NPs can introduce a built-in magnetic field, with little impact on the crystal structure and band structure. The built-in 

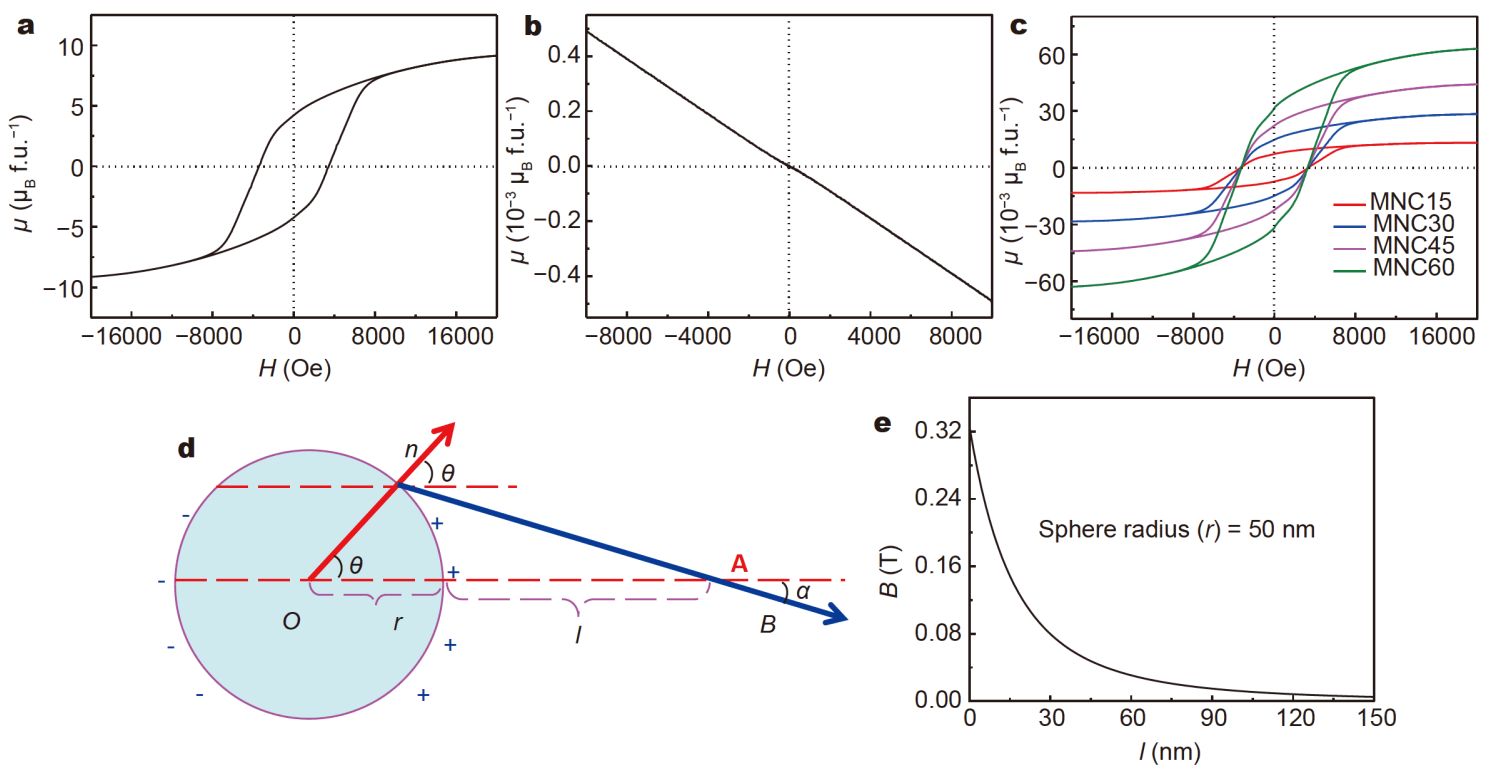

Figure 3 Magnetic properties of BaM-NPs, BiTeSe matrix, and $x \mathrm{BaM}-\mathrm{NPs} / \mathrm{BiTeSe}$ thermoelectromagnetic nanocomposites. Magnetization versus magnetic field $(M-H)$ curves for (a) BaM-NPs, (b) BiTeSe matrix and (c) all thermoelectromagnetic nanocomposites at room temperature. (d) Schematic of magnetic field calculation around a nanoparticle. (e) Magnetic induction intensity versus the distance (l) between the observation point and the nanoparticle.

magnetic field around BaM-NPs is calculated assuming a nanosphere model (Fig. 3d). The magnetic field $B$ at a point " $A$ " can be calculated using the following formula:

$B=\frac{2 J r^{3}}{3(l+r)^{3}}$,

where $J$ is the saturated magnetic polarization intensity of $\mathrm{BaM}, l$ is the distance from the sphere surface, and $r$ is the radius of the sphere.

Assuming that the $r$ of BaM-NPs is about $50 \mathrm{~nm}$ based on STEM and the $J$ is $0.49 \mathrm{~T}$, the maximal built-in magnetic field at BaM-NPs surface is roughly $0.33 \mathrm{~T}$ (Fig. 3e), which decreases to $0.01 \mathrm{~T}$ at $l=100 \mathrm{~nm}$. Additionally, BaM-NPs do not react with the BiTeSe matrix to form a complex interfacial structure or defects as confirmed by STEM. This provides a simple scenario to study how electron transport properties respond as the carriers interact with the built-in magnetic field generated by randomly distributed BaM-NPs. For a moving carrier (electron), the Lorentz force exerted by the built-in magnetic field can bend its trajectory and impact the transport properties.

\section{TE properties of $x \mathrm{BaM}-\mathrm{NPs} / \mathrm{BiTeSe}$}

Fig. 4 displays the electrical transport properties of $x \mathrm{BaM}$ $\mathrm{NPs} / \mathrm{BiTeSe}$ thermoelectromagnetic nanocomposites. The electrical conductivity $(\sigma)$ of all samples monotonously decreases with increasing temperature (Fig. 4a), indicating a metal-like or degenerate semiconductor behavior [18]. As more BaM-NPs are added to the BiTeSe matrix, the $\sigma$ decreases for the measured temperature range. As the $\sigma$ is proportional to the carrier concentration $(n)$ and Hall mobility $\left(\mu_{\mathrm{H}}\right)$, two parameters were used to understand the electrical transport properties. An anomalous Hall effect from BaM-NPs was eliminated by linear fitting of the Hall voltage measured near the saturation magnetic field (0.4-1.2 T) (Fig. S2) [25]. Compared with the BiTeSe matrix, the $n$ of all the nanocomposites gradually decreases with increasing BaM-NPs content (Fig. 4b). For example, the $n$ for the nanocomposite with $x=0.6 \mathrm{wt} \%$ is $6.9 \times 10^{19} \mathrm{~cm}^{-3}$ at $300 \mathrm{~K}$, which shows a $16 \%$ decrease as compared with that of the BiTeSe matrix $(8.2 \times$ $\left.10^{19} \mathrm{~cm}^{-3}\right)$. It has been widely known that doping and interface reaction in the nanocomposites can influence the $n$ [36]. However, atomic-resolution STEM characterization results unambiguously confirm that BaMNPs do not interact with the BiTeSe matrix, indicating that point defects are unlikely to form at the interface. Moreover, XRD results suggest that the lattice constants of BiTeSe do not alter in the nanocomposites, and therefore the band gap is not responsible for the change of $n$. It has also been suggested that the p-n junction can form between the matrix and NPs of opposite carrier type, which can reduce the $n$. However, both the BaM- 

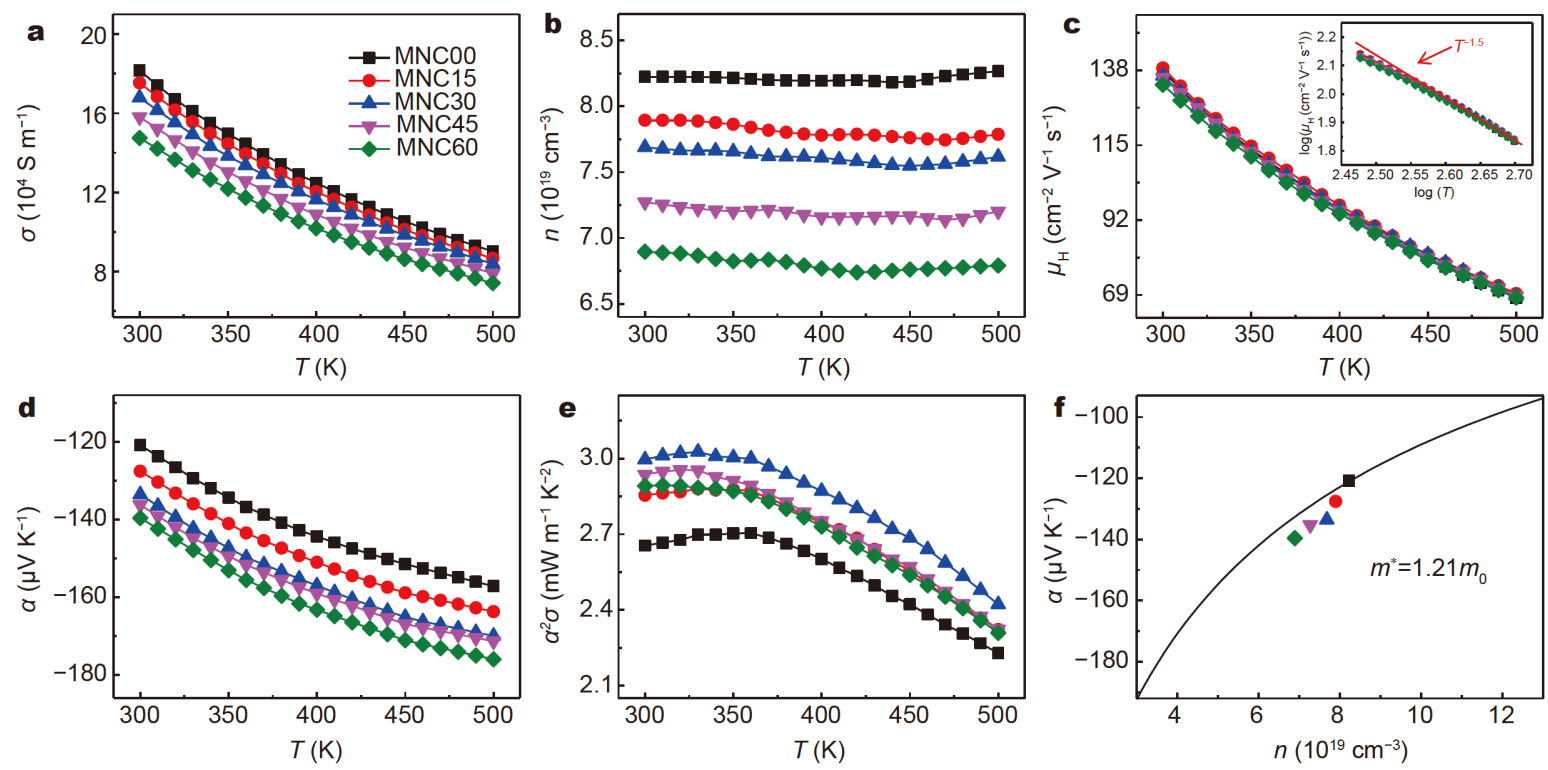

Figure 4 Electrical properties of $x \mathrm{BaM}-\mathrm{NPs} / \mathrm{BiTeSe}$ thermoelectromagnetic nanocomposites. Temperature dependence of (a) electrical conductivity, (b) carrier concentration, (c) hall mobility, (d) Seebeck coefficient, and (e) power factor of $x$ BaM-NPs/BiTeSe $(x=0,0.15,0.30,0.45$ and $0.60 \mathrm{wt} \%)$ thermoelectromagnetic nanocomposites. The inset in (c) is the $\log \left(\mu_{\mathrm{H}}\right)$ versus $\log (T)$. (f) The Seebeck coefficient versus carrier concentration of matrix at $300 \mathrm{~K}$ obtained by the Pisarenko relation using $m^{*}=1.21 m_{0}$.

NPs [37] and BiTeSe matrix are n-type semiconductors, and the depletion of carriers near the interface is not the case here. Therefore, the decrease of $n$ in the nanocomposites is not caused by the interfacial reaction, band structure, or $\mathrm{p}-\mathrm{n}$ junction. A very likely explanation comes from the nanoscale built-in magnetic field induced by BaM-NPs.

In recent years, it has been shown that the nanoscale built-in magnetic field from magnetic NPs in TE materials can significantly influence the transport properties [24-26]. For example, the strong ferromagnetic BaM-NPs in $\mathrm{Ba}_{0.3} \mathrm{In}_{0.3} \mathrm{Co}_{4} \mathrm{Sb}_{12}$ can trap electrons below the Curie temperature of BaM-NPs and release them above the Curie temperature, providing a novel way to regulate the electrical and thermal transport properties [24]. A similar result had been subsequently reported in half-Heusler alloys [26]. The main reason for such behavior is that the nanoscale built-in magnetic field can bend carrier's trajectory due to the Lorentz force, and sometimes the carrier can be trapped around the magnetic field. Above the Curie temperature of magnetic BaM-NPs, the built-in magnetic field disappears and the trapped carriers are released. In the working temperature of $\mathrm{Bi}_{2} \mathrm{Te}_{3}$-based alloys (less than $500 \mathrm{~K}$ ), BaM-NPs stay at the ferromagnetic state (Curie temperature is about $673 \mathrm{~K} \mathrm{[24])} \mathrm{and} \mathrm{thus} \mathrm{the}$ trapping effect always plays a role. The magneto-trapped carrier effect induced by BaM-NPs causes the simulta- neous decrease in the $n$ and $\sigma$ of the nanocomposites. This approach is especially useful for n-type BiTeSe because lowering the carrier concentration is one of the main challenges.

For all the nanocomposites and BiTeSe matrix, the $\mu_{\mathrm{H}}$ values are almost constant (Fig. 4c), because the decrease of $n$ reduces the probability of collision between carriers. The smooth interface between the BiTeSe matrix and BaM-NPs (Fig. 2f) is beneficial to carrier transport. Further, the scattering mechanism can be understood by fitting $\log \left(\mu_{\mathrm{H}}\right)$ versus $\log (T)$, as shown in the inset of Fig. 4c. The $\log \left(\mu_{\mathrm{H}}\right)$ is proportional to $T^{-1.5}$ in the whole temperature range, indicating that the dominant carrier scattering mechanism is acoustic phonon scattering.

The Seebeck coefficient $(\alpha)$ values of all the samples over the measured temperature range are negative, indicating the major carriers in the BiTeSe matrix and BaM-NPs/BiTeSe nanocomposites are electrons (i.e., ntype conduction behavior) (Fig. $4 \mathrm{~d}$ ). The $\alpha$ values of all samples gradually increase with temperature, mainly due to the increased electron scattering from the lattice vibration at high temperatures. However, the intrinsic excitation that normally degrades $\alpha$ is not observed in our cases. Compared with the matrix, the addition of BaMNPs enhances the $\alpha$ over the whole temperature range. At $300 \mathrm{~K}$, the $\alpha$ of matrix is only $121 \mu \mathrm{V} \mathrm{K}^{-1}$, which increases to $140 \mu \mathrm{V} \mathrm{K}^{-1}$ for the nanocomposite with $0.6 \% \mathrm{BaM}$ - 
NPs, a roughly $16 \%$ enhancement. According to Mott equation, the increase in $\alpha$ is usually related to the $n$ or the scattering parameter $(r)$. The decrease of $n$ due to the magneto-trapped carrier effect clearly has a positive effect on the increase of $\alpha$, while how the $r$ changes in the nanocomposites can be understood using the single parabolic band (SPB) model [38]:

$\alpha=-\frac{k_{\mathrm{B}}}{\mathrm{e}}\left(\frac{(r+5 / 2) F_{r+3 / 2}(\eta)}{(r+3 / 2) F_{r+1 / 2}(\eta)}-\eta\right)$,

$n=\frac{4 \pi\left(2 m^{*} k_{\mathrm{B}} T\right)^{3 / 2}}{h^{3}} F_{1 / 2}(\eta)$,

with Fermi integral order of $i$

$F_{i}(\eta)=\int_{0}^{\infty} \frac{\chi^{i}}{1+\mathrm{e}^{\chi^{-\eta}}} \mathrm{d} \chi$,

$\eta=\frac{E_{\mathrm{F}}}{k_{\mathrm{B}} T}$,

where $r$ is the scattering parameter, $E_{\mathrm{F}}$ is the Fermi energy, $\eta$ is the reduced Fermi level, and $F_{i}(\eta)$ is the FermiDirac integral. Assuming acoustic phonon scattering $(r=$ $-0.5)$ is the main scattering mechanism for the BiTeSe matrix, the effective mass $\left(m^{*}=1.21 m_{0}\right)$ of the BiTeSe matrix at $300 \mathrm{~K}$ can be calculated using Equations (2)-(5) and parameters listed in Table 1. The $r$ values of all the nanocomposites are then calculated assuming that the $m^{*}$ is a fixed value (Table 1). It can be seen that all the nanocomposites have larger $r$ than that of the BiTeSe matrix and the nanocomposite with $0.3 \%$ BaM-NPs show the highest $r$, indicating that BaM-NPs can improve the electron scattering. The dependence of $n$ on $\alpha$ can be visualized using the Pisarenko plot as shown in Fig. 4f, calculated using the SPB model with $m^{*}$ of the BiTeSe matrix at room temperature. If there is no additional electron scattering in BaM-NPs/BiTeSe nanocomposites, the $\alpha$ should lie on the Pisarenko plot. In this case, the enhancement of electron scattering moves all the data points below the Pisarenko plot. Therefore, the improvement in $\alpha$ originates from both the decrease of $n$ and the enhanced electron scattering induced by BaMNPs.

As a result, the power factors $\left(\alpha^{2} \sigma\right)$ of all BaM-NPs/ BiTeSe nanocomposites are larger than that of the BiTeSe matrix (Fig. 4e). For the sample with $0.30 \%$ BaM-NPs, the highest $\alpha^{2} \sigma$ value reaches $3.03 \mathrm{~mW} \mathrm{~m}^{-1} \mathrm{~K}^{-2}$ at $330 \mathrm{~K}$. However, for those samples with BaM-NP contents higher than $0.3 \%$, the $\sigma$ continues to deteriorate and the power factor starts to decrease.

All $x \mathrm{BaM}-\mathrm{NPs} / \mathrm{BiTeSe}$ nanocomposites have much lower thermal conductivity $\kappa$ than the BiTeSe matrix over the entire measured temperature range, indicating that BaM-NPs can effectively decrease the thermal conductivity (Fig. 5a). The mechanism can be interpreted from two aspects: the carrier thermal conductivity $\left(\kappa_{\mathrm{E}}\right)$ and lattice thermal conductivity $\left(\kappa_{\mathrm{L}}\right)$. The $\kappa_{\mathrm{E}}$ describes the contribution of carriers on the thermal conductivity, and is described by Weidemann-Franz law, $\kappa_{\mathrm{E}}=L \sigma T$, where $L$, $\sigma$ and $T$ are the Lorenz number, the electrical conductivity and the absolute temperature, respectively. The Lorenz number can be obtained using the Fermi-Dirac statistics [39]:

$L=\left(\frac{k_{\mathrm{B}}}{\mathrm{e}}\right)^{2}\left\{\frac{(r+7 / 2) F_{r+5 / 2}(\eta)}{(r+3 / 2) F_{r+1 / 2}(\eta)}-\left[\frac{(r+5 / 2) F_{r+3 / 2}(\eta)}{(r+3 / 2) F_{r+1 / 2}(\eta)}\right]^{2}\right\}$,

assuming the scattering factor $r=-0.5$ (Fig. 5b). We find that the $\kappa_{\mathrm{E}}$ (Fig. $5 \mathrm{c}$, inset) gradually decreases with increasing BaM-NP contents, which agrees very well with the change of $\sigma$ and $n$. The magneto-trapped carrier effect induced by BaM-NPs has still beneficial influence on decreasing the carrier thermal conductivity.

The $\kappa_{\mathrm{L}}$ describes the contribution from phonon scattering, and is simply the difference between $\kappa$ and $\kappa_{\mathrm{E}}$. For $x \mathrm{BaM}-\mathrm{NPs} / \mathrm{BiTeSe}$, the $\kappa_{\mathrm{L}}$ decreases as more BaM-NPs are added until $x$ reaches $0.45 \%$ (Fig. $5 \mathrm{c}$ ), indicating that BaM-NPs embedded into the BiTeSe matrix can enhance the phonon scattering and reduce the $\kappa_{\mathrm{L}}$. However, MNC60 sample has a $\kappa_{\mathrm{L}}$ slightly larger than that of the BiTeSe matrix. A possible explanation is attributed to the

Table 1 Hall coefficient, Hall mobility, carrier concentration, electrical conductivity, Seebeck coefficient and scattering parameter of $x$ BaM-NPs/ $\operatorname{BiTeSe}(x=0,0.15,0.30,0.45$ and $0.60 \mathrm{wt} \%)$ thermoelectromagnetic nanocomposites at room temperature

\begin{tabular}{|c|c|c|c|c|c|c|}
\hline Samples & $R_{\mathrm{H}}\left(10^{-1} \mathrm{~cm}^{3} \mathrm{C}^{-1}\right)$ & $\mu_{\mathrm{H}}\left(\mathrm{cm}^{2} \mathrm{~V}^{-1} \mathrm{~s}^{-1}\right)$ & $n\left(10^{19} \mathrm{~cm}^{-3}\right)$ & $\sigma\left(10^{4} \mathrm{~S} \mathrm{~m}^{-1}\right)$ & $\alpha\left(\mu \mathrm{V} \mathrm{K}^{-1}\right)$ & $r$ \\
\hline MNC00 & -0.76 & 137.89 & 8.22 & 18.16 & -120.89 & -0.50 \\
\hline MNC15 & -0.79 & 138.69 & 7.89 & 17.54 & -127.57 & -0.44 \\
\hline MNC30 & -0.81 & 136.29 & 7.69 & 16.79 & -133.60 & -0.38 \\
\hline MNC45 & -0.86 & 135.71 & 7.27 & 15.81 & -136.29 & -0.40 \\
\hline MNC60 & -0.91 & 133.57 & 6.90 & 14.75 & -139.63 & -0.40 \\
\hline
\end{tabular}



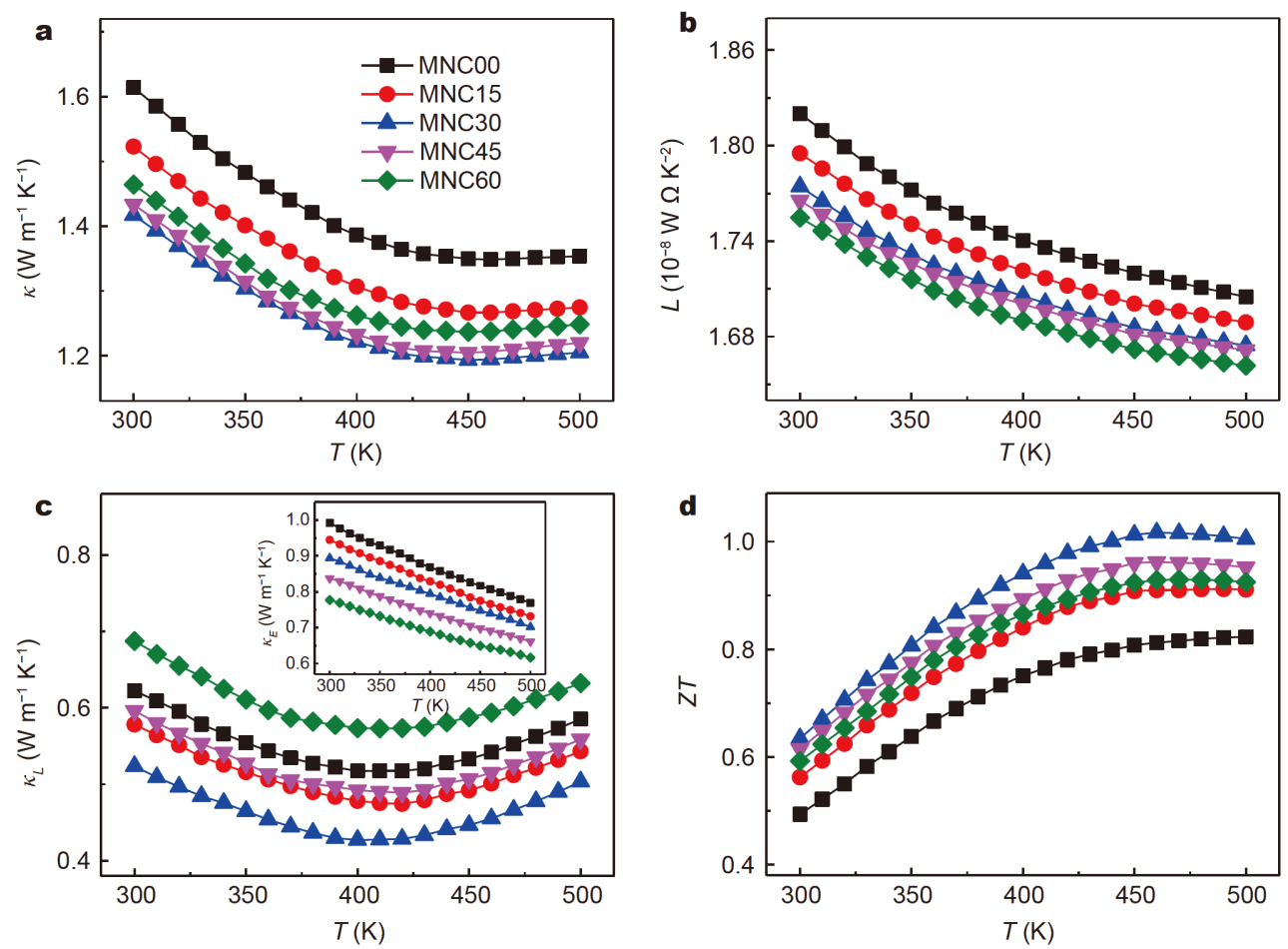

Figure 5 Thermal properties and $Z T$ values of $x \mathrm{BaM}-\mathrm{NPs} / \mathrm{BiTeSe}$ thermoelectromagnetic nanocomposites. Temperature dependence of (a) total thermal conductivity, (b) Lorentz number, (c) lattice thermal conductivity, and (d) $Z T$ for $x \mathrm{BaM}-\mathrm{NPs} / \mathrm{BiTeSe}(x=0.0,0.15,0.30,0.45$ and $0.60 \mathrm{wt} \%)$ thermoelectromagnetic nanocomposites. The inset in (c) shows the temperature dependence of the carrier thermal conductivity.

large intrinsic lattice thermal conductivity of BaM-NPs. As temperature increases, enhanced lattice vibration leads to rapid decrease in $\kappa_{\mathrm{L}}$ for all the samples. As the temperature further increases (over $420 \mathrm{~K}$ ), the thermal transport of the composites is dominated by bipolar thermal diffusion in the intrinsic excitation region, resulting in a slight increase in $\kappa_{\mathrm{L}}$. As a result, the decrease in $\kappa$ for all the nanocomposites is attributed to the significant decrease of $\kappa_{\mathrm{E}}$ and $\kappa_{\mathrm{L}}$. The lowest $\kappa$ is $1.19 \mathrm{~W} \mathrm{~m}^{-1} \mathrm{~K}^{-1}$ at $460 \mathrm{~K}$ for the MNC30 sample.

The $Z T$ values of all the samples are calculated with the measured $\alpha, \sigma$ and $\kappa$ (Fig. 5d). In general, thermoelectromagnetic nanocomposites have a larger $Z T$ than the matrix due to the reduction of $\kappa$ and/or the enhancement of $\alpha^{2} \sigma$. A maximum $Z T$ value reaches 1.0 at $460 \mathrm{~K}$ for the MNC30 sample, a $25 \%$ increase compared with that of the BiTeSe matrix. The magneto-trapped carrier effect induced by BaM-NPs eventually leads to high $Z T$ values due to the simultaneous decreases in the carrier concentration and the thermal conductivity.

\section{Cooling performance of $x \mathrm{BaM}-\mathrm{NPs} / \mathrm{BiTeSe}$}

To further evaluate the role of the magneto-trapped carrier effect in enhancing the electro-thermal conversion performance, two single-leg devices with same dimension of $2.5 \mathrm{~mm} \times 3.5 \mathrm{~mm} \times 11 \mathrm{~mm}$ were fabricated using matrix (MNC00) and 0.3\% BaM-NPs/BiTeSe (MNC30), which are named MNC00D and MNC30D, respectively. The heat-side temperature $\left(T_{\mathrm{H}}\right)$ and cool-side temperature $\left(T_{\mathrm{C}}\right)$ were measured for different working currents (I) (Fig. 6). Generally, $T_{\mathrm{H}}$ rapidly increases and $T_{\mathrm{C}}$ rapidly decreases until equilibrium is obtained over time. The cooling temperature difference $\left(\Delta T_{\mathrm{C}}\right)$ between the coolside temperature and room temperature $\left(T_{\mathrm{R}}\right)$ is calculated with $\Delta T_{\mathrm{C}}=T_{\mathrm{R}}-T_{\mathrm{C}}$. The working temperature difference $(\Delta T)$ between $T_{\mathrm{C}}$ and $T_{\mathrm{H}}$ is calculated with $\Delta T=T_{\mathrm{H}}-T_{\mathrm{C}}$. $\Delta T_{\mathrm{C}}$ and $\Delta T$ were used to evaluate the cooling performance of the two single-leg devices. As the $I$ increases from 0.5 to $2.5 \mathrm{~A}$, the $\Delta T_{\mathrm{C}}$ firstly increases due to the Peltier effect and then decreases due to the increasing Joule heat while the $\Delta T$ gradually increases, which is further verified by infrared imaging detection (Fig. S3). The maximum $\Delta T_{\mathrm{C}}$ of MNC30D and MNC00D are obtained at $I=1.5 \mathrm{~A}$ and $I=1.0 \mathrm{~A}$, respectively. The maximum $\Delta T_{\mathrm{C} 30}$ of MNC30D reaches $3.8 \mathrm{~K}$, increased by $65 \%$ as compared with $2.3 \mathrm{~K}$ of MNC00D. This result clearly shows that the magneto-trapped carrier effect induced by BaM-NPs in $x$ BaM-NPs/BiTeSe thermo- 

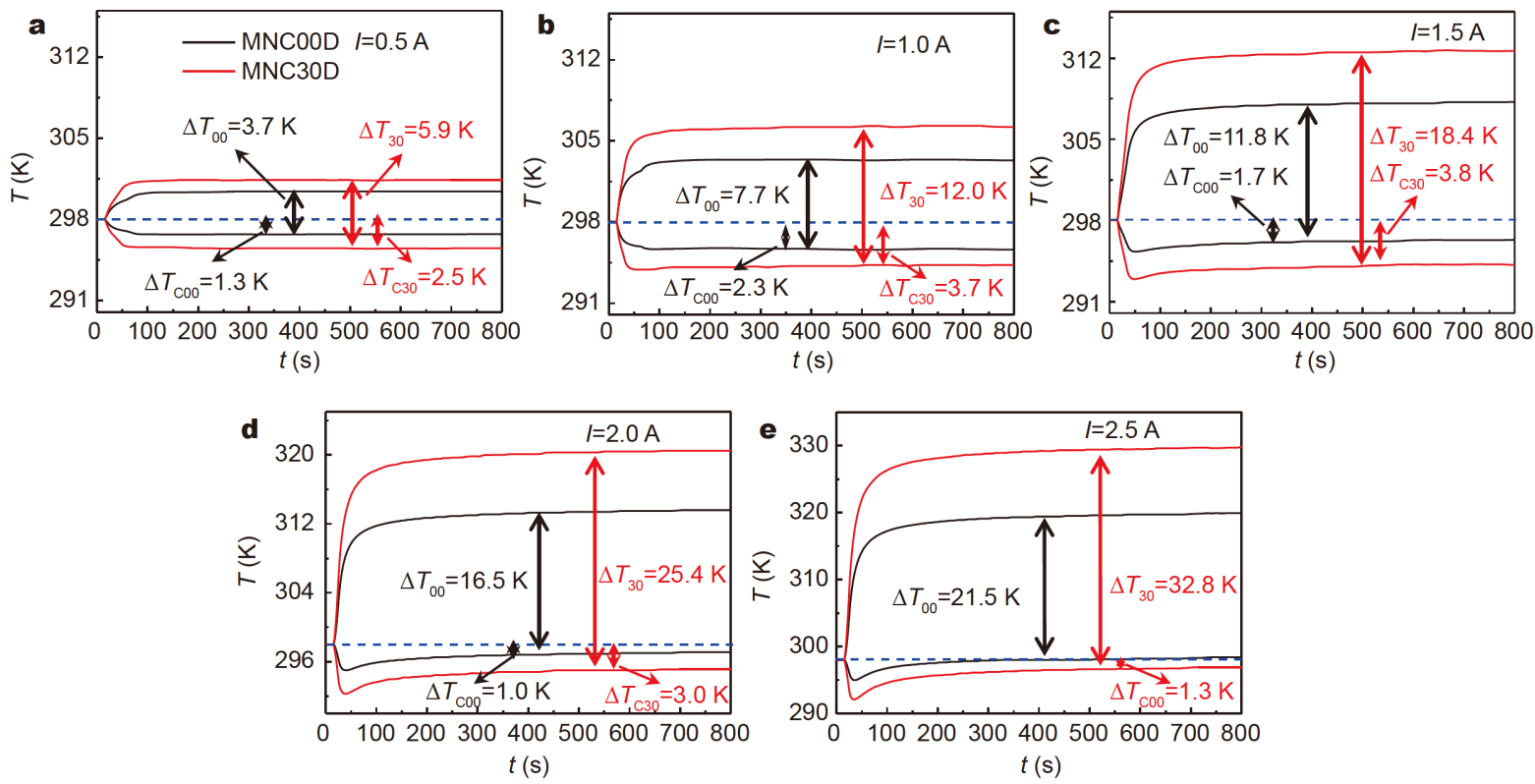

Figure 6 Effect of BaM-NPs on cooling performance. (a-e) Cooling performances of MNC00D (black) and MNC30D (red) under different operating currents.

electromagnetic nanocomposites can remarkably enhance the cooling performance.

\section{CONCLUSIONS}

In summary, this work has demonstrated that incorporating hard-magnetic BaM-NPs in a series of BaM$\mathrm{NPs} / \mathrm{BiTeSe}$ thermoelectromagnetic nanocomposites leads to synergistical regulation of electron and phonon transport properties on the nanoscale. The novel magneto-trapped carrier effect induced by BaM-NPs is discovered to reduce the carrier concentration by $16 \%$ and increase the Seebeck coefficient by $16 \%$. At the same time, the phonon scattering centers provided by BaM-NPs reduce the thermal conductivity by $12 \%$. Due to the combined effect, the $Z T$ value of the nanocomposites is enhanced by more than $25 \%$ in the range of $300-450 \mathrm{~K}$, and the cooling temperature difference increased by $65 \%$ near room temperature. This work shows that the magneto-trapped carrier effect induced by hard-magnetic NPs has great potential in enhancing room-temperature electro-thermal conversion performance of TE materials with high carrier concentrations.

Received 19 February 2021; accepted 15 March 2021; published online 12 May 2021

1 Bell LE. Cooling, heating, generating power, and recovering waste heat with thermoelectric systems. Science, 2008, 321: 1457-1461

2 Zhu H, Xiao C, Xie Y. Design of highly efficient thermoelectric materials: Tailoring reciprocal-space properties by real-space modification. Adv Mater, 2018, 30: 1802000

3 Zhao W, Wei P, Zhang Q, et al. Enhanced thermoelectric performance in barium and indium double-filled skutterudite bulk materials via orbital hybridization induced by indium filler. J Am Chem Soc, 2009, 131: 3713-3720

4 Shi X, Yang J, Salvador JR, et al. Multiple-filled skutterudites: High thermoelectric figure of merit through separately optimizing electrical and thermal transports. J Am Chem Soc, 2011, 133: 7837-7846

5 Zhao W, Wei P, Zhang Q, et al. Multi-localization transport behaviour in bulk thermoelectric materials. Nat Commun, 2015, 6: 6197

6 Sun B, Zhao J, Li Y, et al. Effect of HPHT processing on structural and thermoelectric properties of low-cost type-I clathrate $\mathrm{Ba}_{8} \mathrm{Cu}_{6}-$ $\mathrm{Si}_{40}$. J Phys Chem C, 2020, 124: 9082-9088

7 Heremans JP, Jovovic V, Toberer ES, et al. Enhancement of thermoelectric efficiency in $\mathrm{PbTe}$ by distortion of the electronic density of states. Science, 2008, 321: 554-557

8 Pei Y, Shi X, LaLonde A, et al. Convergence of electronic bands for high performance bulk thermoelectrics. Nature, 2011, 473: 66-69

9 Zhu T, Liu Y, Fu C, et al. Compromise and synergy in highefficiency thermoelectric materials. Adv Mater, 2017, 29: 1605884

10 Chang $\mathrm{C}, \mathrm{Wu} \mathrm{M}, \mathrm{He} \mathrm{D}$, et al. 3D charge and 2D phonon transports leading to high out-of-plane $Z T$ in n-type SnSe crystals. Science, 2018, 360: 778-783

11 Biswas K, He J, Blum ID, et al. High-performance bulk thermoelectrics with all-scale hierarchical architectures. Nature, 2012, 489: $414-418$

12 Mao J, Zhu H, Ding Z, et al. High thermoelectric cooling performance of n-type $\mathrm{Mg}_{3} \mathrm{Bi}_{2}$-based materials. Science, 2019, 365: 495498

13 Ren GK, Lan JL, Zhao LD, et al. Layered oxygen-containing 
thermoelectric materials: Mechanisms, strategies, and beyond. Mater Today, 2019, 29: 68-85

14 Hu L, Zhu T, Liu X, et al. Point defect engineering of high-performance bismuth-telluride-based thermoelectric materials. Adv Funct Mater, 2014, 24: 5211-5218

15 Zhu B, Liu X, Wang Q, et al. Realizing record high performance in n-type $\mathrm{Bi}_{2} \mathrm{Te}_{3}$-based thermoelectric materials. Energy Environ Sci, 2020, 13: 2106-2114

16 Witting IT, Chasapis TC, Ricci F, et al. The thermoelectric properties of bismuth telluride. Adv Electron Mater, 2019, 5: 1800904

17 Poudel B, Hao Q, Ma Y, et al. High-thermoelectric performance of nanostructured bismuth antimony telluride bulk alloys. Science, 2008, 320: 634-638

18 Pan Y, Wei TR, Wu CF, et al. Electrical and thermal transport properties of spark plasma sintered n-type $\mathrm{Bi}_{2} \mathrm{Te}_{3-x} \mathrm{Se}_{x}$ alloys: The combined effect of point defect and Se content. J Mater Chem C, 2015, 3: 10583-10589

19 Deng R, Su X, Hao S, et al. High thermoelectric performance in $\mathrm{Bi}_{0.46} \mathrm{Sb}_{1.54} \mathrm{Te}_{3}$ nanostructured with ZnTe. Energy Environ Sci, 2018, 11: 1520-1535

20 Hong M, Chasapis TC, Chen ZG, et al. n-type $\mathrm{Bi}_{2} \mathrm{Te}_{3-x} \mathrm{Se}_{x}$ nanoplates with enhanced thermoelectric efficiency driven by widefrequency phonon scatterings and synergistic carrier scatterings. ACS Nano, 2016, 10: 4719-4727

$21 \mathrm{Wu}$ Y, Yu Y, Zhang Q, et al. Liquid-phase hot deformation to enhance thermoelectric performance of n-type bismuth-telluridebased solid solutions. Adv Sci, 2019, 6: 1901702

$22 \mathrm{Hu} \mathrm{L}, \mathrm{Wu} \mathrm{H}, \mathrm{Zhu} \mathrm{T}$, et al. Tuning multiscale microstructures to enhance thermoelectric performance of n-type bismuth-telluridebased solid solutions. Adv Energy Mater, 2015, 5: 1500411

$23 \mathrm{Hu} \mathrm{L}$, Guo Y, Li J, et al. Control of donor-like effect in $\mathrm{V}_{2} \mathrm{VI}_{3}$ polycrystalline thermoelectric materials. Mater Res Bull, 2018, 99: 377-384

24 Zhao W, Liu Z, Wei $\mathrm{P}$, et al. Magnetoelectric interaction and transport behaviours in magnetic nanocomposite thermoelectric materials. Nat Nanotech, 2017, 12: 55-60

25 Zhao W, Liu Z, Sun Z, et al. Superparamagnetic enhancement of thermoelectric performance. Nature, 2017, 549: 247-251

26 Lu R, Lopez JS, Liu Y, et al. Coherent magnetic nanoinclusions induce charge localization in half-Heusler alloys leading to high- $T_{\mathrm{c}}$ ferromagnetism and enhanced thermoelectric performance. J Mater Chem A, 2019, 7: 11095-11103

27 Tsujii N, Nishide A, Hayakawa J, et al. Observation of enhanced thermopower due to spin fluctuation in weak itinerant ferromagnet. Sci Adv, 2019, 5: eaat5935

28 Li J. Unexpected boost of thermoelectric performance by magnetic nanoparticles. Sci China Mater, 2017, 60: 1023-1024

29 Wang $\mathrm{H}$, Luo X, Chen W, et al. Magnetic-field enhanced highthermoelectric performance in topological Dirac semimetal $\mathrm{Cd}_{3} \mathrm{As}_{2}$ crystal. Sci Bull, 2018, 63: 411-418

30 Kim G, Kim HS, Lee HS, et al. Synchronized enhancement of thermoelectric properties of higher manganese silicide by introducing Fe and Co nanoparticles. Nano Energy, 2020, 72: 104698

31 Li C, Ma S, Wei P, et al. Magnetism-induced huge enhancement of the room-temperature thermoelectric and cooling performance of p-type BiSbTe alloys. Energy Environ Sci, 2020, 13: 535-544

32 Ma S, Li C, Wei P, et al. High-pressure synthesis and excellent thermoelectric performance of $\mathrm{Ni} / \mathrm{BiTeSe}$ magnetic nanocomposites. J Mater Chem A, 2020, 8: 4816-4826

33 Xiao C, Li K, Zhang J, et al. Magnetic ions in wide band gap semiconductor nanocrystals for optimized thermoelectric properties. Mater Horiz, 2014, 1: 81-86

34 Ge ZH, Zhang YX, Song D, et al. Excellent $Z T$ achieved in $\mathrm{Cu}_{1.8} \mathrm{~S}$ thermoelectric alloys through introducing rare-earth trichlorides. J Mater Chem A, 2018, 6: 14440-14448

35 Mansfield R. The magnetic susceptibility of bismuth telluride. Proc Phys Soc, 1959, 74: 599-603

36 Huang ZY, Zhang H, Yang L, et al. Achieving high thermoelectric performance of $\mathrm{Ni} / \mathrm{Cu}$ modified $\mathrm{Bi}_{0.5} \mathrm{Sb}_{1.5} \mathrm{Te}_{3}$ composites by a facile electroless plating. Mater Today Energy, 2018, 9: 383-390

37 Ravinder D, Shalini P, Mahesh P, et al. High temperature thermoelectric power studies of cobalt and titanium substituted barium hexagonal ferrites. J Magn Magn Mater, 2004, 268: 154-158

38 Liu Z, Geng H, Shuai J, et al. The effect of nickel doping on electron and phonon transport in the n-type nanostructured thermoelectric material CoSbS. J Mater Chem C, 2015, 3: 10442 10450

39 Liu WS, Zhang Q, Lan Y, et al. Thermoelectric property studies on $\mathrm{Cu}$-doped n-type $\mathrm{Cu}_{x} \mathrm{Bi}_{2} \mathrm{Te}_{2.7} \mathrm{Se}_{0.3}$ nanocomposites. Adv Energy Mater, 2011, 1: 577-587

Acknowledgements This work was supported by the National Natural Science Foundation of China (11834012, 51620105014, 91963207, 91963122 and 51902237), the National Key Research and Development Program of China (2018YFB0703603, 2019YFA0704900 and SQ2018YFE010905), and Foshan Xianhu Laboratory of Advanced Energy Science and Technology Guangdong Laboratory (XHT2020-004). XRD, FESEM, EPMA and HRTEM experiments were performed at the Center for Materials Research and Testing of Wuhan University of Technology. The S/TEM work was performed at the Nanostructure Research Center (NRC), which is supported by the Fundamental Research Funds for the Central Universities (WUT: 2021III016GX). The authors thank Meijun Yang and Chunhua Shen for their help in the structural characterization.

Author contributions Ma S performed the experiments; Sang X performed the TEM microstructure analyses on the samples; Ma $S$ and Sang X wrote and revised the manuscript with input of all authors; Wei P, Zhu W, Nie X, Sun FH and Zhang Q participated in the discussion of the results and the paper planning; Zhao $\mathrm{W}$ and Sang $\mathrm{X}$ directed this project.

Conflict of interest The authors declare that they have no conflict of interest.

Supplementary information Supporting data are available in the online version of the paper.

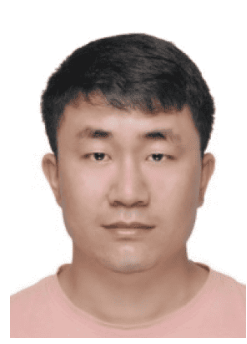

Shifang Ma received his MSc degree from Shenyang Jianzhu University in 2016. Currently, he is pursuing his $\mathrm{PhD}$ at the State Key Laboratory of Advanced Technology for Materials Synthesis and Processing (SKLATMSP) of Wuhan University of Technology (WUT), China. His research interest focuses on the thermoelectric bulk materials. 


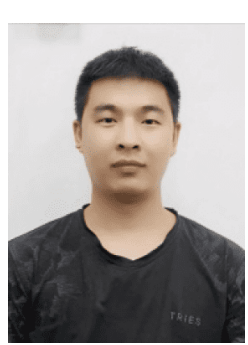

Cuncheng $\mathbf{L i}$ is a $\mathrm{PhD}$ candidate at SKLATMSP of WUT, China. He received his BSc degree from Shandong University of Science and Technology in 2015. His research mainly focuses on bismuth telluride and nanocomposite thermoelectric materials.

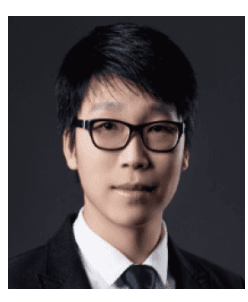

Xiahan Sang is a professor at WUT. He received a BE degree from Wuhan University in 2005, a Master's degree from the Institute of Metal Research, Chinese Academy of Sciences in 2008, and obtained his $\mathrm{PhD}$ in materials science from the University of Pittsburgh in 2012. His research focuses on the microstructure characterization of thermo-electric-magnetic materials, and the development of novel quantitative TEM techniques.

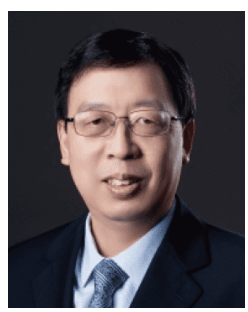

Wenyu Zhao received his MSc degree in 1996 at China University of Geosciences and $\mathrm{PhD}$ degree in 2004 at WUT. He has been working at SKLATMSP of WUT as a full professor since 2008. His research interests include thermoelectric materials, magnetic thermoelectric materials, magnetic ferrite materials, novel structure thermoelectric devices, and thermoelectric application technology.

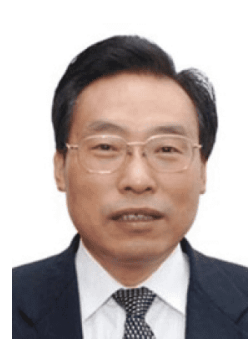

Qingjie Zhang received his MSc degree in 1984 and $\mathrm{PhD}$ degree in 1990 from Huazhong University of Science and Technology. Currently, he is an academician of the Chinese Academy of Sciences, the president of WUT, and director of SKLATMSP. His research focuses on the thermoelectric materials and the related application technology.

\section{磁增强电热转换性能}

马世方 ${ }^{1,2 \dagger}$, 李存成 ${ }^{1 \dagger}$, 崔文俊 ${ }^{1,3}$, 桑夏唅 ${ }^{1,3^{*}}$, 魏平 ${ }^{1,3}$, 朱婉婷 ${ }^{1}$, 聂晓蕾 ${ }^{1}$, 孙富华 ${ }^{1,2}$, 赵文俞 ${ }^{1 *}$, 张清态 $^{1}$

摘要 在同一尺度协同调控非笼状结构热电材料载流子输运和声 子输运面临巨大挑战. 本文设计并制备了 $\mathrm{BaFe}_{12} \mathrm{O}_{19} / \mathrm{Bi}_{2} \mathrm{Te}_{2.5} \mathrm{Se}_{0.5}$ 热 电磁纳米复合材料, 实现了纳米尺度协同调控电热输运性能. 研究 表明, $\mathrm{BaFe}_{12} \mathrm{O}_{19}$ 硬磁纳米粒子产生的磁束缚载流子效应导致载流 子浓度降低 $16 \%$, Seebeck系数提高 $16 \%$. 同时, $\mathrm{BaFe}_{12} \mathrm{O}_{19}$ 纳米粒子 作为声子散射中心, 增强了声子散射, 使热导率降低 $12 \%$. 热电磁纳 米复合材料在 300-450 K温度范围内的平均 $Z T$ 值提高了 $25 \%$, 室温 附近的制冷温差提高了 $65 \%$. 本研究极大地拓宽了 $n$ 型 $\mathrm{Bi}_{2} \mathrm{Te}_{2.5} \mathrm{Se}_{0.5}$ 的商业化应用潜力, 证明了磁束缚载流子效应是一种提升高载流 子浓度热电材料电热转换性能的普适方法. 